\title{
Cooling of neutron stars with stiff stellar matter*
}

\author{
H. Grigorian ${ }^{1,2}$, D. N. Voskresensky ${ }^{3}$, D. Blaschke B $^{3,4,5}$ \\ ${ }^{1}$ Laboratory of Information Technologies, JINR, RU-141980 Dubna \\ ${ }^{2}$ Department of Theoretical Physics, Yerevan State University, AM-0025 Yerevan \\ ${ }^{3}$ National Research Nuclear University (MEPhI), RU-115409 Moscow \\ ${ }^{4}$ Institute of Theoretical Physics, Wroclaw University, PL-50-204 Wroclaw \\ ${ }^{5}$ Bogoliubov Laboratory of Theoretical Physics, JINR, RU-141980 Dubna
}

\begin{abstract}
Recent evidence for high masses $\left(\sim 2 M_{\odot}\right)$ pulsars PSR J1614-2230 and PSR J0348-0432 requires neutron star matter to have a stiff equation of state (EoS). The thermal evolution of compact stars (CS) with stiff hadronic EoS necessitates the application of the "nuclear medium cooling" scenario with a selection of appropriate proton gap profiles together with in-medium effects (like pion softening) on cooling mechanisms in order to achieve a satisfactory explanation of all existing observational data for the temperature-age relation of CS. Here we focus on two examples from [1] for a stiff hadronic EoS without (DD2 EoS) and with (DD2vex) excluded volume correction.
\end{abstract}

PACS numbers: 97.60.Jd,95.30.Cq,26.60-c

\section{Introduction}

The neutrino emissivities of various processes taking place in deeper layers of compact stellar objects are depending on the behavior of the nucleonnucleon $(N N)$ interaction and the proton and neutron paring gaps as functions of the density. An information about these cooling processes, the heat transport and the EoS of compact star (CS) matter can be gained from analyzing the data on the evolution of surface temperatures of CS.

The available data (see Fig. 3 below) can be separated into three groups related to slow cooling objects (labeled $8 ; 5 ; 1 ; 2 ; 4$; A in Fig. 3), intermediate cooling $(3 ; 6 ; 7$; Cas A; B; E) and rapid cooling $(\mathrm{C} ; \mathrm{D})$ objects. In order

* Presented at the International Conference on Critical Point and Onset of Deconfinement (CPOD'2016), May, 29 - June, 5, 2016, University of Wroclaw 
to explain the difference in the cooling of the slowly and rapidly cooling objects a three order of magnitude difference in their luminosities is required. Therefore, one needs to take into account the strong density-dependence (and thus neutron star (NS) mass dependence) of the medium modification of the $N N$ interaction. Otherwise it is not easy to appropriately explain the essentially different surface temperatures of various objects in the hadronic scenario within the so called "minimal cooling paradigm", cf. [2, where the only relevant rapid process is the so called pair-breaking-formation (PBF) process on neutrons paired in the $3 P_{2}$ channel.

The main medium modification is caused by the softening of the pion exchange contribution with increasing density for $n \gtrsim n_{0}$, where $n_{0} \simeq 0.16$ $\mathrm{fm}^{-3}$ is the nuclear saturation density, and by the density dependent superfluid pairing gaps, see [3, 4] for details. So the cooling of various sources should be essentially different due to the difference in their masses. This effect of course strongly depends on the density profiles of star structure and thus on the EoS. The recent measurements of the high masses of the pulsars PSR J1614-2230 [5, 6] and PSR J0348-0432 [7] on the one hand and of the low masses for PSR J0737-3039B [8] for the companion of PSR J1756-2251 [9, 10] on the other have provided the proof for the existence of CS with masses varying at least from 1.2 to $2.0 M_{\odot}$. It requires a relatively stiff EoS to reach the high value of the mass on the stable branch of CS. On the other hand for a stiff EoS lower densities are reached in the cores of stars with the same mass compared to those for a soft EoS.

\section{EoS}

The HHJ hadronic EoS, which has been exploited in our previous works, was stiffened in [12] for $n>4 n_{0}$ to comply with the constraint that the EoS should allow for a maximum NS mass above the value $M=2.01 \pm 0.04 M_{\odot}$ measured for PSR J0348+0432 by [7], see also [5, 6]. However, the resulting EoS (labeled as HDD), which produces $M_{\max }=2.06 M_{\odot}$, still might be not sufficiently stiff, since the existence of even more massive objects than those known up to now [5, 6, 7] is not excluded. In Fig. 11 we show the mass central density and mass - radius relationship for different EoS models used in our simulations of cooling evolution of CS.

\section{Effective pion gap and $p p$ pairing gaps}

The most efficient processes within the hadronic "nuclear medium cooling" scenario, cf. [4, are the medium modified Urca (MMU) processes, like $n n \rightarrow n p e \bar{\nu}$, i.e. the modified Urca (MU) processes computed by taking into account pion softening effects [3] (see Fig. 2 left), and the PBF processes $N \rightarrow N_{\text {pair }} \nu \bar{\nu}, N=n$ or $p$. While being enhanced owing to their 


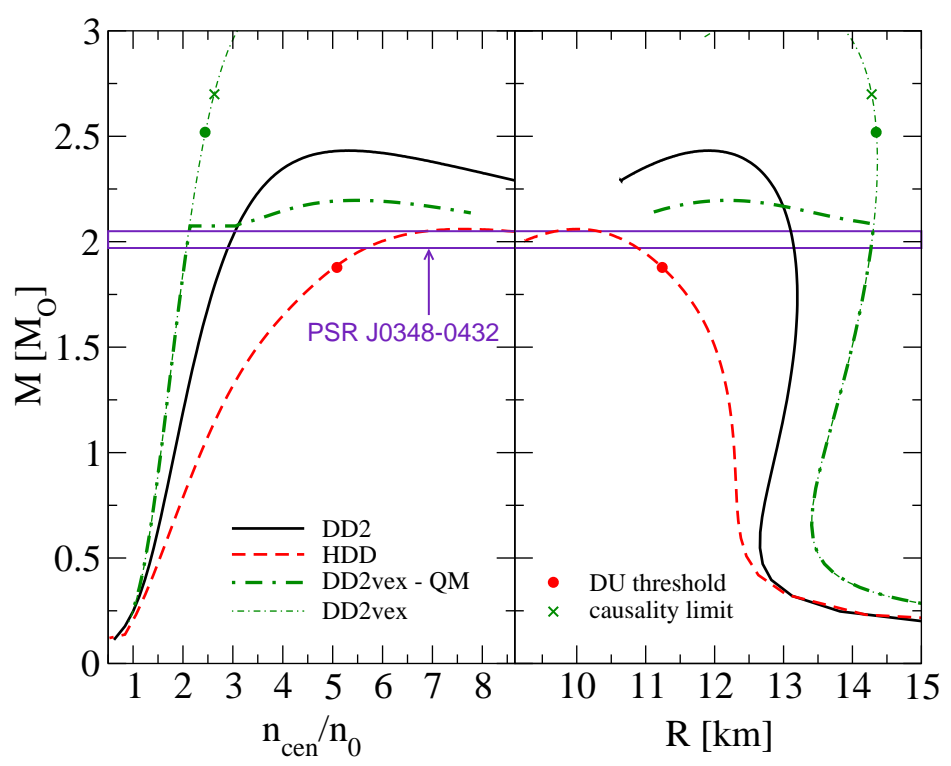

Fig. 1. Mass vs. central baryon density (left) and radius (right) for the HDD (dash lines), a stiffer DD2 [11] (solid lines) and a still stiffer DD2vex hadronic EoS (dash-dotted lines). Bold dash-dotted continuations show a possible quark (QM) transition. The crosses for the DD2vex hadronic EoS indicate violation of the causality. The band shows the data on the measured mass for the pulsar PSR J0348-0432. The bold dots indicate the DU thresholds on hadronic branches.

one-nucleon nature the latter processes are allowed only in the presence of nucleon pairing and should be computed by taking into account in-medium effects in the weak interaction vertices. Various parametrizations for the effective pion gap being exploited here are shown in Fig. 2 left.

Note that in the minimal cooling scenario one assumes that the emissivity of the PBF process on protons is suppressed by two orders of magnitude compared to that for the PBF process on neutrons since the authors use the free $p \rightarrow p \nu \bar{\nu}$ vertices, whereas in matter the decay may occur through the neutron and neutron hole and the electron and electron hole in the intermediate states of the reaction, cf. [4]. Note [3] that the contribution of the intermediate reaction states is the largest in the emissivity of the MMU processes for $n \gtrsim n_{0}$. This contribution is not incorporated at all in the MU emissivity used in the minimal cooling scheme.

The resulting cooling curves are rather insensitive to the values of the 

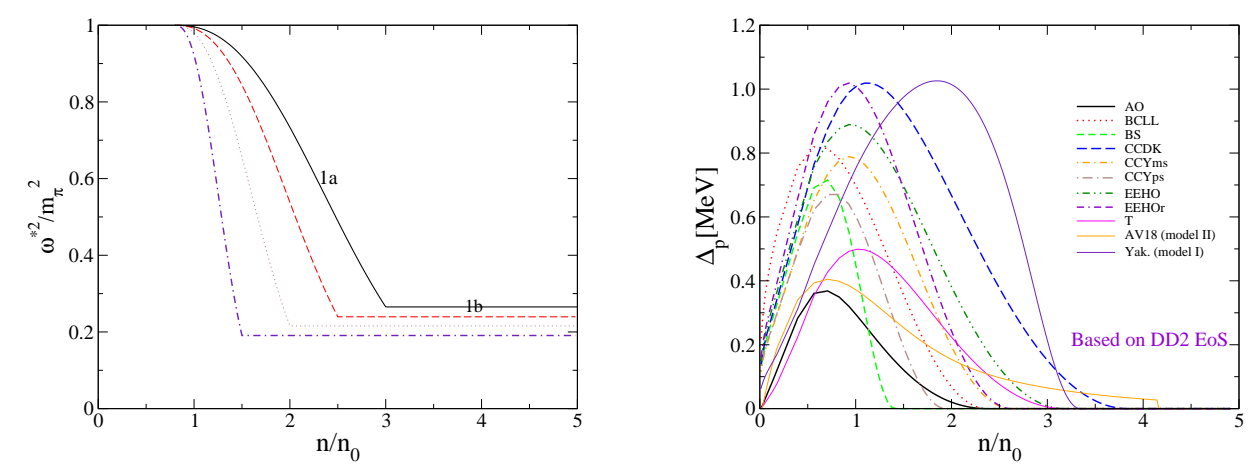

Fig. 2. Left panel: Square of the effective pion gap as a function of the density (curves labeled a), with a pion softening saturated above a critical value $n_{c}^{\pi}$ (i.e., disregarding a possibility of a pion condensation), see lines labeled b, $m_{\pi}$ is the pion mass. The solid line $(1 \mathrm{a}+1 \mathrm{~b}), n_{c}^{\pi}=3 n_{0}$, corresponds to the same parametrization as in 13 , the other lines, $n_{c}^{\pi}=2.5 n_{0}, 2 n_{0}$ and $1.5 n_{0}$ demonstrate a stronger pion softening effect. Right panel: $1 S_{0} p p$ gaps as functions of the baryon density for zero temperature. The abbreviations in the legend correspond to those used in Ref. 14. The gaps labeled as "Yak" and "AV18" are those (models I and II, respectively) exploited in our previous works [13, 15, 16, 12] (right panel).

$1 S_{0} n n$ pairing gaps but sensitive to the choice of the $1 S_{0} p p$ gaps and $3 P_{2}$ $n n$ gaps, since $1 S_{0} n n$ pairing gaps drop already for $n \gtrsim 0.6-0.8 n_{0}$, whereas $1 S_{0} p p$ gaps are spreading to $n \sim 1.5-4 n_{0}$, and $3 P_{2} n n$ gaps may spread to a higher density, see Fig. 2 right panel. Indeed, the dense interior rather than the crust determines the total luminosity within our scenario. The gaps are sensitive to in-medium ( $N N$ loop) effects, and their values are badly known due to exponential dependence on the $N N$ interaction amplitude in the pairing channel. Especially the values of the $3 P_{2}$ neutron pairing gaps are poorly known. Ref. [17] computed a tiny value of $\Delta\left(3 P_{2}\right) \lesssim 10 \mathrm{keV}$. In our model an overall fit of the CS cooling data is obtained for a strongly suppressed value of the $3 P_{2}$ neutron pairing gap, thus being in favour of this results. The dependence of the cooling curves on the $3 P_{2}$ neutron and $1 S_{0}$ proton paring gaps was studied within our scenario in [15]. The successful description of all cooling data within our scenario, where many in-medium effects are shown to be important while they are being disregarded in the minimal cooling scenario, demonstrates that the statement made within the latter scenario in some works, that an appropriate fit of existing Cas A data allows to "measure" the critical temperature of the $3 P_{2} n n$ pairing as $T_{c} \sim(5-9) \cdot 10^{8} \mathrm{~K}$, is not justified. Note that the authors of the recent paper 14] have explained the Cas A ACIS-S data for the NS mass $M=1.44 M_{\odot}$ 
within the minimal cooling scenario using the stiff BSk21 EoS, a large proton gap and a moderate $3 P_{2}$ neutron gap. Hottest and coldest objects, however, can hardly be explained appropriately within the same scenario.
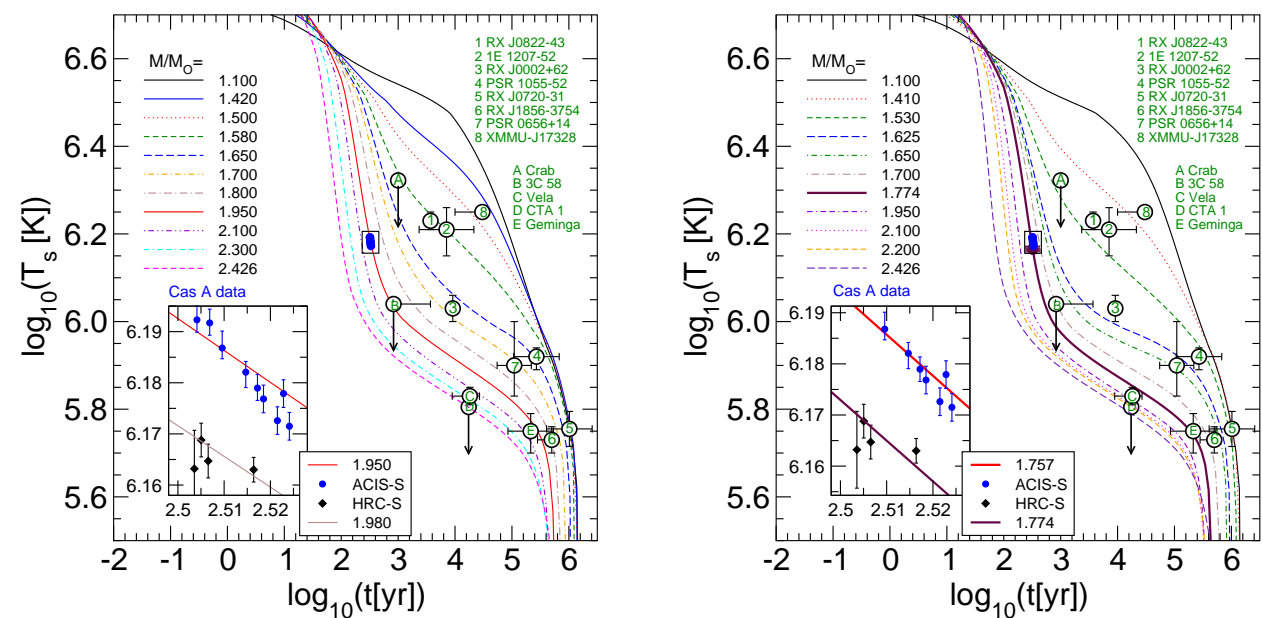

Fig. 3. Cooling curves for a NS sequence according to the hadronic DD2 EoS; $n_{c}^{\pi}=3 n_{0}$, EEHOr (left) and $n_{c}^{\pi}=2.5 n_{0}$, CCYms models (right), see the text.
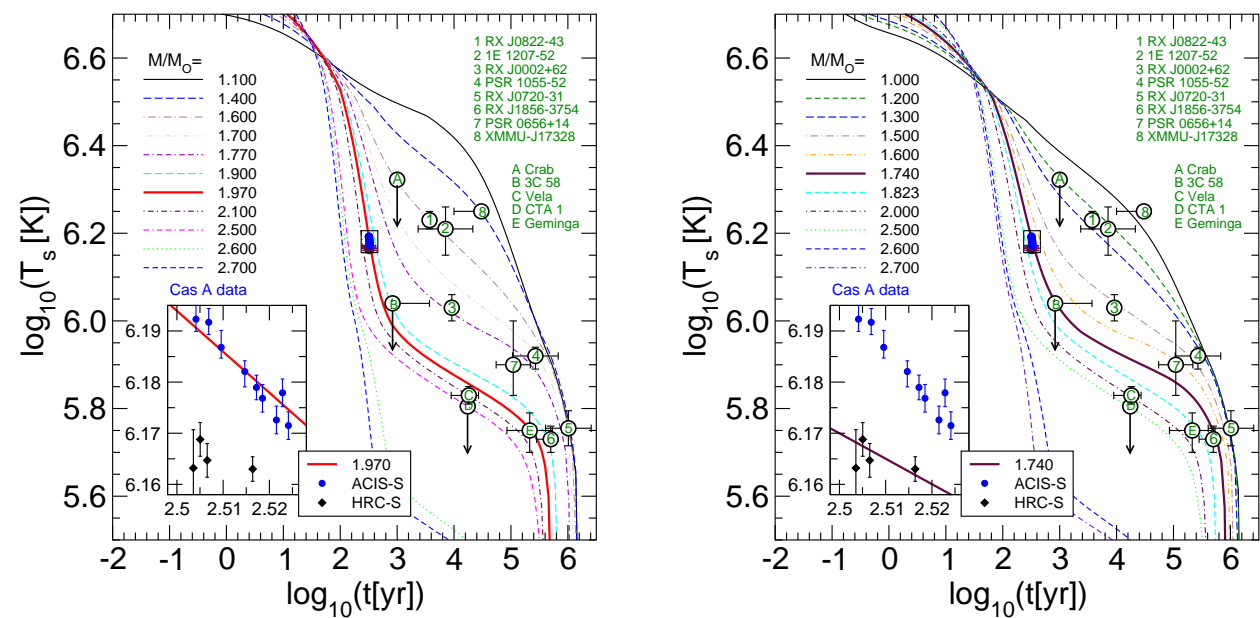

Fig. 4. Cooling curves for a NS sequence according to the hadronic DD2vex EoS; $n_{c}^{\pi}=2.5 n_{0}$, BCLL (left) and AO (right) $p p$ pairing models, for details see the text. 


\section{Cooling}

In Fig. 3 we demonstrate the CS surface temperature $T_{s}$ and age $t$ relation. In the left panel we present results for the effective pion gap given by the solid curve $1 \mathrm{a}+1 \mathrm{~b}$ in Fig. 2 left, $n_{c}^{\pi}=3 n_{0}$. The $1 S_{0} p p$ pairing gap corresponds to model EEHOr. In the right panel we exploit the dashed curve of Fig. 2 left, $n_{c}^{\pi}=2.5 n_{0}$, and $1 S_{0} p p$ pairing gap model CCYms (cf. Fig. 2 right panel). The mass range is shown in the legend. Comparison with Cas A ACIS-S and HRC-S data is shown in the inset. The cooling data for Cas A from the ACIS-S instrument are explained by a NS mass $M=1.950 M_{\odot}\left(\right.$ those of the HRC-S instrument by $\left.M=1.980 M_{\odot}\right)$, and $M=1.757 M_{\odot}\left(\right.$ and $\left.M=1.774 M_{\odot}\right)$ correspondingly.

The CS surface temperature $T_{s}$ vs. age $t$ for the model EoS DD2vex is shown in the Fig. 4 left and right panels. The effective pion gap corresponds to $n_{c}^{\pi}=2 n_{0}$, the dotted curve a+b in Fig. 2 left. In the left panel the $1 S_{0}$ $p p$ pairing gap corresponds to the model BCLL (Fig. 2 right panel). In the right panel the $1 S_{0} p p$ pairing gap corresponds to the model AO (Fig. 2 right panel). Cas A cooling data from the ACIS-S and HRC-S instruments are explained with a NS of $M=1.970 M_{\odot}$ and $M=1.740 M_{\odot}$, correspondingly.

\section{Conclusions}

If in the future a very massive compact star (CS), and/or a CS of a middle mass but with a large radius would be observed, this would provide arguments for a stiff equation of state (EoS), like DD2 or even DD2vex, see Fig. 1. Both EoS that we used, DD2 and DD2vex, are compatible with the existing CS cooling data provided we exploit the nuclear medium cooling scenario developed in our previous works, under the assumption that different sources have different masses. The resulting cooling curves prove to be sensitive to the value and the density dependence of the $p p$ pairing gap and the effective pion gap. Both ACIS-S and HRC-S data for Cas A can be fitted with the help of a variation of the model parameters. A stiffer EoS suggests a stronger pion softening effect.

Another consequence of stiff hadronic EoS which was not elaborated in this contribution is a phase transition to deconfined quark matter at low densities but high mass, as shown in Fig. 1. If the stable hybrid star branch of such models could be populated in nature, it would add another strip of cooling curves in the temperature-age diagram, either separated from the hadronic cooling curves discussed here or overlapping with them, depending on the characteristics of the cooling processes in quark matter. In this context it is interesting to discuss the cooling of high-mass twin stars [18, 19] which, if they would exist in nature, populate a separate branch of stable hybrid stars, disconnected from the hadronic one. The discovery 
of these objects would imply the existence of a critical endpoint in the QCD phase diagram [20, 21]. The investigation of their role for CS cooling scenarios is an important task which we take up in subsequent work.

\section{Acknowledgments}

This work was supported by the NCN Opus programme under contract UMO-2014/13/B/ST9/02621 and by the Ministry of Education and Science of the Russian Federation (Basic part). We acknowledge the support from the MEPhI Academic Excellence Project under contract number 02.a03.21.0005 and from the Bogoliubov-Infeld and Ter-Antonian-Smorodinsky programmes as well as from the COST Action MP1304 "NewCompStar".

\section{REFERENCES}

[1] H. Grigorian, D. N. Voskresensky and D. Blaschke, Eur. Phys. J. A 52, no. 3, 67 (2016).

[2] K. G. Elshamouty, et al., Astrophys. J. 777, 22 (2013).

[3] A. B. Migdal, E. E. Saperstein, M. A. Troitsky and D. N. Voskresensky, Phys. Rept. 192, 179 (1990).

[4] D. N. Voskresensky, Lect. Notes Phys. 578, 467 (2001).

[5] P. Demorest, et al., Nature 467, 1081 (2010).

[6] E. Fonseca et al., Astrophys. J. 832, no. 2, 167 (2016).

[7] J. Antoniadis, et al., Science 340, 6131 (2013).

[8] M. Kramer, et al., Science 314, 97 (2006).

[9] A. J. Faulkner, et al., Astrophys. J. 618, L119 (2004).

[10] R. D. Ferdman et al., Mon. Not. Roy. Astron. Soc. 443, no. 3, 2183 (2014).

[11] S. Typel, et al., Phys. Rev. C81, 015803 (2010).

[12] D. Blaschke, H. Grigorian and D. N. Voskresensky, Phys. Rev. C88, 065805 (2013).

[13] D. Blaschke, H. Grigorian and D. N. Voskresensky, Astron. Astrophys. 424, 979 (2004).

[14] W. C. G. Ho, et al., Phys. Rev. C 91, 015806 (2015).

[15] H. Grigorian and D. N. Voskresensky, Astron. Astrophys. 444, 913 (2005).

[16] D. Blaschke, et al., Phys. Rev. C 85, 022802 (2012).

[17] A. Schwenk and B. Friman, Phys. Rev. Lett. 92, 082501 (2004).

[18] S. Benic, et al., Astron. Astrophys. 577, A40 (2015).

[19] D. Alvarez-Castillo, et al., Eur. Phys. J. A 52, no. 3, 69 (2016).

[20] D. Blaschke, D. E. Alvarez-Castillo and S. Benic, PoS CPOD 2013, 063 (2013).

[21] D. Alvarez-Castillo, et al., Eur. Phys. J. A 52, no. 8, 232 (2016). 